\title{
Palmitate-triggered COX2/PGE2-mediated hyperinflammation in dual-stressed PdL fibroblasts is mediated by repressive H3K27 trimethylation.
}

\author{
Lisa Schuldt 1, Michael Reimann 2, Katrin von Brandenstein 1, Julia Steinmetz 1, Annika Döding 2, \\ Ulrike Schulze-Späte ${ }^{2}$, Collin Jacobs ${ }^{3}$ and Judit Symmank ${ }^{1, *}$ \\ 1 Department of Orthodontics, University Hospital Jena, Leutragraben 3, 07743 Jena, Germany \\ 2 Section of Geriodontics, Department of Conservative Dentistry and Periodontics, University Hospital Jena, \\ Leutragraben 3, 07743 Jena, \\ 3 Department of Orthodontics, Center for Dental, Oral and Maxillofacial Medicine, University Hospital Jena, \\ An der Alten Post 4, 07743 Jena, Germany \\ * Correspondence: judit.symmank@med.uni-jena.de
}

\begin{abstract}
The interrelationship between periodontal disease, obesity-related hyperlipidemia and mechanical forces as well as their modulating effects on the epigenetic profile of periodontal ligament (PdL) cells are assumed to be remarkably complex. The PdL serves as connective tissue between teeth and alveolar bone for pathogen defense and inflammatory response to mechanical stimuli occurring during tooth movement. Altered inflammatory signaling could promote root resorption and tooth loss. Hyperinflammatory COX2/PGE2 signaling was reported for human PdL fibroblasts (HPdLF) concomitantly stressed with $P$. gingivalis lipopolysaccharides and compressive force after exposure to palmitic acid (PA). The aim of this study was to investigate to what extent this is modulated by global and gene-specific changes in histone modifications. Quantitative expression of epigenetic key players and global H3Kac as well as H3K27me3 levels were evaluated in dual stressed HPdLF exposed to PA revealing a decreased force-related reduction in repressive H3K27me3. UNC1999-induced H3K27me3 inhibition reversed the hyperinflammatory response of dual-stressed PA-cultures characterized by COX2 expression, PGE2 secretion and THP1 adhesion. Reduced expression of the anti-inflammatory cytokine IL10 and increased association of H3K27me3 at its promoter-associated sites were reversed by inhibitor treatment. Thus, the data highlight an important epigenetic interplay between different stimuli to which the PdL is exposed.
\end{abstract}

Keywords: Periodontitis, Tooth movement, Obesity, Palmitic Acid, Histone Modification, Inflammation, COX2/PGE2, IL10

\section{Introduction}

Genetic predisposition, unfavorable environmental conditions and an unhealthy lifestyle are important risk factors for the onset and progression of periodontal disease, but cannot fully account for individual susceptibility [1]. In view of the high prevalence of this chronic, non-communicable disease and subsequent economic health care system implications [2], the investigation of additional key regulatory mechanisms has been in focus of recent periodontitis research. This has drawn attention to epigenetic regulatory mechanisms as important factors in the pathogenesis of the disease [3]. Epigenetic modifications include in part heritable changes on DNA and histones that restrict the accessibility of DNA to the transcription machinery without altering the genome [4]. With regard to the inflammatory aspects of periodontal disease, of these alterations, DNA methylation and post-translational modifications (PTM) to histone tail amino acids have been thoroughly investigated [5]. PTMs at histones include acetylation and methylation, which modulate transcriptional activity in a context-dependent manner [6]. While the attachment of acetyl groups by histone acetyltransferases (HAT) is generally associated with an 
opening of chromatin structure and a beneficial environment for gene expression, the effects of histone methylation depend on the appropriately modified amino acid and the abundance of methyl groups [6,7]. For example, trimethylation of lysines at position 27 (K27) on histone $3(\mathrm{H} 3)$ has been linked to transcriptional repression, while H3K4 trimethylation is associated with gene activation [7]. Regarding the expression of key factors regulating PTMs, a number of comparative studies showed differences between periodontal patients and healthy subjects [5], presumably caused by the pathogenic infection and possibly linked to an excessive presence of bacterial metabolites such as short-chain fatty acids [8].

Elevated serum levels of long-chain fatty acids such as saturated palmitic acid (PA) are typically associated with obesity and have been investigated for its role in diseaserelated low-grade systemic inflammation [9-11]. Further, a pro-inflammatory characteristic of PA has been shown under hyperlipidemic conditions [12-20], although in general fatty acids are relevant for normal cell functions [21]. Several studies reported an interrelationship between both diseases. For example, a delayed response to the infection with gram-negative oral anaerobe Porphyromonas gingivalis ( $P$. gingivalis) was reported for obese mice [22]. P. gingivalis has been described as a keystone pathogen affecting oral health and disease, possibly due to its unique ability to evade the host immune response [23]. It has been demonstrated that a variety of virulence factors such as lipopolysaccharides (LPS), gingipains and fimbriae are responsible for its pathogenity [24]. In animal studies, enhanced swallowing of $P$. gingivalis was reported to induce changes in gut microbiome, increase systemic inflammation and induce metabolic alterations [25,26]. In addition, adipocytes stimulated with $P$. gingivalis-LPS exhibited a more pronounced pro-inflammatory profile, indicating a potential contributing factor to the pro-inflammatory state associated with obesity $[27,28]$. Although various changes in the host oral environment and bacterial biofilm composition are required for the onset and progression of periodontitis, the pro-inflammatory impact of $P$. gingivalis-LPS is commonly used to mimic periodontitis-causing conditions in vitro.

Even though inflammatory processes are increased in both diseases, their concomitant impact on the inflammatory response of the periodontal ligament (PdL) to mechanical forces such as during trauma, mastication or orthodontic tooth movement is still poorly studied. The PdL is the connective tissue between teeth and alveolar bone, and its most abundant cell type, PdL fibroblasts, modulate the transient, aseptic inflammatory response to compressive and tensile forces in a temporal and spatial manner [29-31]. Alterations in their inflammatory response may increase the risk of tooth root resorption and tooth loss, primarily by affecting tissue and bone remodeling cells [32,33]. In rats suffering from periodontitis, up-regulated expression of several cytokines in the PdL and enhanced presence of activated osteoclasts were observed after tooth movement in addition to an increased extent of dorsal root resorption [34]. However, an additional impact of obesityrelated changes on tooth movement has not yet been investigated in either animal or human studies. In view of the tremendous heterogeneity of obesity-associated cellular adaptions, in vivo studies can be quite challenging to conduct. Thus, even the results of prospective controlled clinical trials examining only the effects of increased BMI on orthodontic tooth movement (OTM) are conflicting, reporting both increased and decreased rates of OTM [35-38].

However, influences on epigenetic regulatory mechanisms are evident with regards to high-fat diets and obesity-associated alterations [39]. In this line, increased histone acetylation was reported in several cell types under hyperlipidemic conditions, potentially due to the role of fatty acids as lipid-based alternative donors for acetyl-CoA that is required for this epigenetic mark [21,40]. In addition, a potential influence of PA on repressive methylated histone marks has recently been reported [41,42]. This could provide a framework for the interplay with periodontitis-induced changes and a target for potential therapeutic interventions, as epigenetic inhibitors are already widely used in treatment of a variety of diseases [43]. 
We recently reported that with PA incubated human periodontal ligament fibroblasts (HPdLF) showed excessive inflammatory response to simultaneous stimulation with $P$. gingivalis-LPS and compressive force mainly through enhanced secretion of prostaglandin E2 (PGE2) [44], which is regulated by cyclooxygenase 2 (COX2) [45]. The aim of this study was to investigate whether this excessive inflammation is based on epigenetic alterations induced by palmitic acid.

\section{Materials and Methods}

\subsection{Cell culture}

Culture medium consisting of Dulbecco's modified Eagle medium (DMEM; Thermo Fisher Scientific, Carlsbad, CA, USA) containing $4.5 \mathrm{~g} / \mathrm{L}$ glucose, 10\% heat-inactivated fetal bovine serum (Thermo Fisher Scientific, Carlsbad, CA, USA),), $100 \mathrm{U} / \mathrm{mL}$ penicillin, $100 \mu \mathrm{g} / \mathrm{mL}$ streptomycin, and $50 \mathrm{mg} / \mathrm{L}$ L-ascorbic acid were used for growing commercially acquired human periodontal ligament fibroblast (HPdLF, Lonza, Basel, Switzerland) at $37 \circ \mathrm{C}, 5 \% \mathrm{CO} 2$ and 95\% humidity. HPdLF were passaged at a confluency of 75\% with $0.05 \%$ Trypsin/EDTA (Thermo Fisher Scientific, Carlsbad, CA, USA) and used for experiments at passage four to eight.

RPMI 1640 medium (Thermo Fisher Scientific, Carlsbad, CA, USA) containing 10\% FBS, $100 \mathrm{U} / \mathrm{ml}$ penicillin and $100 \mu \mathrm{g} / \mathrm{ml}$ streptomycin was used for culturing THP1 cells (DMSZ, Braunschweig, Germany) at $37^{\circ} \mathrm{C}, 5 \% \mathrm{CO} 2$ and $95 \%$ humidity. Weekly, passages were performed and $1 \times 10^{6}$ cells were seeded into new T175 culture flask for further culturing (Thermo Fisher Scientific, Carlsbad, CA, USA).

\subsection{Stimulation with Palmitic Acid}

For the analysis of RNA expression, $2.5 \times 10^{4} \mathrm{HPdLF}$ were seeded into each well of a 6-well plate. For immunofluorescence staining, $5 \times 10^{3}$ cells were plated onto coverslips into each well of a 48-well-plate. Cells were cultured for $24 \mathrm{~h}$ in DMEM culture medium prior six-day stimulation with $200 \mu \mathrm{M}$ palmitic acid (PA). PA containing media was produced as described previously [44]. Briefly, dried PA was dissolved at $70 \circ \mathrm{C}$ in sterile water containing $50 \mathrm{mM} \mathrm{NaOH}$, complexed with $37{ }^{\circ} \mathrm{C}$ preheated bovine serum albumin (BSA, Seqens IVD, Limoges, France) and added to DMEM culture medium. BSA-containing medium was used for control stimulation.

\subsection{Stimulation with P. gingivalis Lipopolysaccharides}

To simulate pathogenic stimulation, $10 \mu \mathrm{g} / \mathrm{mL}$ lipopolysaccharids (LPS) of Porphyromonas gingivalis ( $P$. gingivalis; InvivoGen, San Diego, CA, USA) were applied to the culture medium for six hours simultaneous to compressive force application.

\subsection{UNC1999 application}

For inhibition of H3K27 trimethylation, different concentrations of the EZH1/EZH2 inhibitor UNC1999 $(0,5 \mu \mathrm{M}, 1.0 \mu \mathrm{M}$ and $1.5 \mu \mathrm{M})$ were applied for 24 hours on $75 \%$ confluent HPdLF. For further treatment 1.5 $\mu \mathrm{M}$ UNC1999 was applied for six hours simultaneous to $P$. ginigivalis LPS and compressive force application.

\subsection{Application of compressive force}

A compressive force of $2 \mathrm{~g} / \mathrm{cm}^{2}$ was applied according to the protocol of Kirschneck et al. [46] and as previously reported [47]. Briefly, simulataneous to LPS application, glass plates were placed on fatty acid cultured HPdLF for six hours at $37{ }^{\circ} \mathrm{C}, 5 \%$ $\mathrm{CO}_{2}$ and $95 \%$ humidity. Afterwards cells were either directly isolated with TRIzol Reagent (Thermo Fisher Scientific, Carlsbad, CA, USA) for expression analysis or with Dulbecco's Phosphate-Buffered Solution (DPBS, Gibco) for histone extraction and subsequently protein analysis. 
In 48-well plates the compressive force was applied via six-hour centrifugation at $30{ }^{\circ} \mathrm{C}$ with a force of $7.13 \mathrm{~g} / \mathrm{cm}^{2}$. Control cells were cultured at $30{ }^{\circ} \mathrm{C}$ for the duration of force application.

\subsection{Expression analysis with quantitative PCR}

RNA isolation, cDNA synthesis and quantitative PCR were performed as previously described [48]. Primer sequences used for target amplification are shown in Table $\mathbf{1 .}$

Table 1: qPCR primer sequences of human genes indicated in 5 -3 direction. bp, base pairs; fw, forward;

Length, amplicon length; PRC, polycomp repressive complex; rev, reverse.

\begin{tabular}{|c|c|c|c|c|}
\hline Gene & Gene Symbol & NCBI Gene ID & Primer Sequence & Length \\
\hline $\begin{array}{l}\text { Prostaglandin-endoperoxide } \\
\text { synthase } 2\end{array}$ & $\begin{array}{l}\text { PTGS2 } \\
(\text { COX2) }\end{array}$ & 5743 & $\begin{array}{l}\text { fw: GATGATTGCCCGACTCCCTT } \\
\text { rev: GGCCCTCGCTTATGATCTGT }\end{array}$ & $185 \mathrm{bp}$ \\
\hline $\begin{array}{l}\text { Embryonic ectoderm } \\
\text { development }\end{array}$ & $E E D$ & 8726 & $\begin{array}{l}\text { fw: TGCGATGGTTAGGCGATTTG } \\
\text { rev: CCAAATGTCACACTGGCTGT }\end{array}$ & $158 \mathrm{bp}$ \\
\hline $\begin{array}{l}\text { Enhancer of zeste } 1 \text { PRC2 } \\
\text { subunit }\end{array}$ & EZH1 & 2145 & $\begin{array}{l}\text { fw: CGAGAATGTGACCCTGACCT } \\
\text { rev: TTATGAAGGTGCCCCATCCG }\end{array}$ & $154 \mathrm{bp}$ \\
\hline $\begin{array}{l}\text { Enhancer of zeste } 2 \text { PRC2 } \\
\text { subunit }\end{array}$ & $E Z H 2$ & 2146 & $\begin{array}{l}\text { fw: ACAGTTCGTGCCCTTGTGTG } \\
\text { rev: CACTCTCGGACAGCCAGGTA }\end{array}$ & $148 \mathrm{bp}$ \\
\hline Histone deacetylase 2 & HDAC2 & 3066 & $\begin{array}{l}\text { fw: ACTGATGCTTGGAGGAGGTG } \\
\text { rev: CTGGAGTGTTCTGGTTTGTCA }\end{array}$ & $185 \mathrm{bp}$ \\
\hline Histone deacetylase 3 & HDAC3 & 8841 & $\begin{array}{l}\text { fw: GCTGGGTGGTGGTGGTTATA } \\
\text { rev: TTCTGATTCTCGATGCGGGT }\end{array}$ & $174 \mathrm{bp}$ \\
\hline Interleukin 10 & IL10 & 3586 & $\begin{array}{l}\text { fw: AGCCATGAGTGAGTTTGACA } \\
\text { rev: AGAGCCCCAGATCCGATTTT }\end{array}$ & $141 \mathrm{bp}$ \\
\hline Lysine acetyltransferase 8 & KAT8 & 84148 & $\begin{array}{l}\text { fw: GCAAGATCACTCGCAACCAA } \\
\text { rev: AGTCTTCGGGGAATGGTGAG }\end{array}$ & $195 \mathrm{bp}$ \\
\hline Nuclear receptor coactivator 3 & NCOA3 & 8202 & $\begin{array}{l}\text { fw: GGCTCTATTCCCACATTGCC } \\
\text { rev: CCCAGTTGGTTAGATGCTGC }\end{array}$ & $158 \mathrm{bp}$ \\
\hline Ribosomal protein L22 & RPL22 & 6146 & $\begin{array}{l}\text { fw: TGATTGCACCCACCCTGTAG } \\
\text { rev: GGTTCCCAGCTTTTCCGTTC }\end{array}$ & $98 \mathrm{bp}$ \\
\hline $\begin{array}{l}\text { SIN3 transcription regulator } \\
\text { family member A }\end{array}$ & $\operatorname{SIN} 3 A$ & 25942 & $\begin{array}{l}\text { fw: GAGCAGCAGGAAAAGGAAGG } \\
\text { rev TGTAGACGCTTGCTTACACG }\end{array}$ & $200 \mathrm{bp}$ \\
\hline TATA-box binding protein & TBP & 6908 & $\begin{array}{l}\text { fw: CGGCTGTTTAACTTCGCTTCC } \\
\text { rev: TGGGTTATCTTCACACGCCAAG }\end{array}$ & $86 \mathrm{bp}$ \\
\hline
\end{tabular}

\subsection{Immunofluorescent staining}

To detect trimethylated H3K27 after specific treatments, coverslips with cultured HPdLF were fixed in 4\% PFA for 10 minutes, washed with phosphate-buffered saline (PBS and incubated with the primary antibody for 1.5 hours and the secondary antibody for 45 minutes. DAPI (Thermo Fisher Scientific, Carlsbad, CA, USA; 1:10000 in PBS) was used for nuclei staining. Following antibodies were used: Mouse-anti-human H3K9/14/18/23/27 (Thermo Fisher Scientific, Carlsbad, CA, USA; 1:500), Rabbit-anti-human H3K27me3 (Thermo Fisher Scientific, Carlsbad, CA, USA; 1:250), Goat-anti-mouse-Cy5 and goat-antiRabbit-Cy5 (Jackson ImmunoResearch, Baltimore Pike, PA, USA; 1:1000).

\subsection{THP1 adhesion assay}

To detected pro-inflammatory cytokine secretion by stimulated HPdLF, THP1 adhesion assay was performed as previously described [48]. Adhesive THP1 cell numbers were determined and displayed in relation to the total number of HPdLF per each image. 


\subsection{MTT assay}

Cell vitality was analyzed at the plate reader Infinite ${ }^{\circledR}$ M Nano (TECAN, Männedorf, Swiss) with the MTT colorimetric assay (Sigma Aldrich, St. Louis, Missouri, USA) according to manufacturer's protocol.

\subsection{Nuclear extraction andhistone methyltransferase activity assay}

EpiQuik Nuclear Extraction Kit (EpiGentek, Farmingdale, New York, USA) was used for nuclear extraction according to manufacturer's protocol. The activities of histone methyltransferases specifically methylating H3K27 were analyzed with EpiQuik Histone Methyltransferase Activity/Inhibition Assay Kit H3K27 (EpiGentek, Farmingdale, New York, USA) according to manufacturer`s guidelines at the plate reader Infinite ${ }^{\circledR}$ M Nano (TECAN, Männedorf, Swiss).

\subsection{ELISA}

Medium supernatant of stimulated HPdLF was isolated 24 hours after force application to ensure proper protein secretion. To analyzed PGE2 secretion, prostaglandin E2 ELISA (PGE2; R\&D Systems, Minneapolis, MN, USA) was used according to the manufacturer`s guidelines.

\subsection{Chromatin-Immunoprecipitation}

To analyze the association of acetylated H3K at IL10 gene regions, chromatin-immunoprecipitation (ChIP) was performed. First, DNA and protein were cross-linked with $1 \%$ formaldehyde in PBS for $10 \mathrm{~min}$ and neutralized with $120 \mathrm{mM}$ glycine. After cell harvesting in ice-cold PBS and centrifugation for $5 \mathrm{~min}$ at $1000 \mathrm{xg}$ at $4^{\circ} \mathrm{C}$, an approximate cell number of $1 \times 10^{6}$ cells per tube was stored at $-80^{\circ} \mathrm{C}$. ChIP was performed with the ZymoSpin ChIP Kit (Zymo Research, Freiburg, Germany) according to manufacturer's protocol with the ChIP-valuated antibodies rabbit-anti-human H327me3 (Thermo Fisher Scientific, Carlsbad, CA, USA) and mouse-anti-IgG (Thermo Fisher Scientific, Carlsbad, CA, USA). Throughout the ChIP process, one percent input controls were stored for later normalization. The amount of specific DNA fragments bound to H3K27me3 was determined as previously described [49]. Briefly, prior quantitative analysis with the qTOWER3 (Analytik Jena, Jena, Germany) according to the manufacturer`s protocol using Luminaris Color HiGreen qPCR Master Mix (Thermo Fisher Scientific, Carlsbad, CA, USA), primer-specific pre-amplification was performed. Primer sequences used for analysis are displayed in Table 2. Per cent-input method was used to normalize DNA content [50]. IgG control was subtracted from H3K27me3-specific sample.

Table 2 qPCR primer pairs located in the human IL10 in promoter regions (\#1, \#2, \#3) and a non-promoter region (\#4) indicated in 5 -3` direction. bp, base pairs; fw, forward; Length, amplicon length; rev, reverse.

\begin{tabular}{lclc}
\hline Location & Label & Primer Sequence & Length \\
promoter region & $\# 1$ & $\begin{array}{l}\text { fw: TGAAGAAGTCCTGATGTCAC } \\
\text { rev: TTACCTATCCCTACTTCCCC }\end{array}$ & $187 \mathrm{bp}$ \\
promoter region & $\# 2$ & $\begin{array}{l}\text { fw: AGCACTACCTGACTAGCATA } \\
\text { rev: AGAGACTGGCTTCCTACAG }\end{array}$ & $192 \mathrm{bp}$ \\
promoter region & $\# 3$ & $\begin{array}{l}\text { fw: GGGGACCCAATTATTCTCA } \\
\text { rev: TGGGCTACCTCTCTTAGAAT }\end{array}$ & $188 \mathrm{bp}$ \\
non-promoter region & $\# 4$ & $\begin{array}{l}\text { fw: GCTTAGAGCGTTTCCAGACC } \\
\text { rev: CTCCCCACTGTAGACATCCA }\end{array}$ & $131 \mathrm{bp}$ \\
\hline
\end{tabular}

\subsection{Microscopy, Image Analysis, and Statistics}

THP1 adhesion assay, H3Kac and H3K27me3 staining was imaged with the inverted confocal laser scanning microscope TCS SP5 (Leica, Wetzlar, Germany). Fiji software (https://imagej.net/Fiji, accessed on 01.04.2017) was used to analyze THP1 cell numbers and fluorescence intensity. Graph Pad Prism (https://www.graphpad.com, accessed on 
01.02.2021) was used for statistical analysis. Adobe Photoshop CS5 (https://adobe.com, accessed on 01.02.2013) was used for figure illustration. One-way ANOVA and post hoc test (Tukey) were used as statistical tests. All experiments were independently repeated at least three times and examined in technical duplicates. Diagrams show mean \pm SE. Sig-

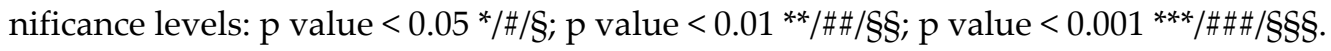

\section{Results}

\subsection{Palmitic acid exposure impacts force-induced reduction of H3K27me3 in LPS-stimulated} human periodontal ligament fibroblasts

Free fatty acids can serve as an alternative source of acetyl CoA and thus affect $\mathrm{H3}$ lysine acetylation of human periodontal ligament fibroblasts [51]. To elucidate the role of H3K acetylation in the regulation of the hyper-inflammatory response of PA-exposed HPdLFs simultaneously stimulated by mechanical and bacterial-induced stress, we first examined the expression of relevant regulators. Quantitative PCR, however, revealed neither changes in genes encoding major histone acetyltransferase (NCOA3, KAT8), nor histone deacetylases (HDAC2, HDAC3) in dual stimulated PA cultures as compared to BSA controls (Fig. 1a). Additional quantitative analysis of immunofluorescently stained H3K9/14/18/23/27 acetylation showed increased levels due to compressive forces, however, no differences due to PA exposure were detected as well (Fig. 1b, c).

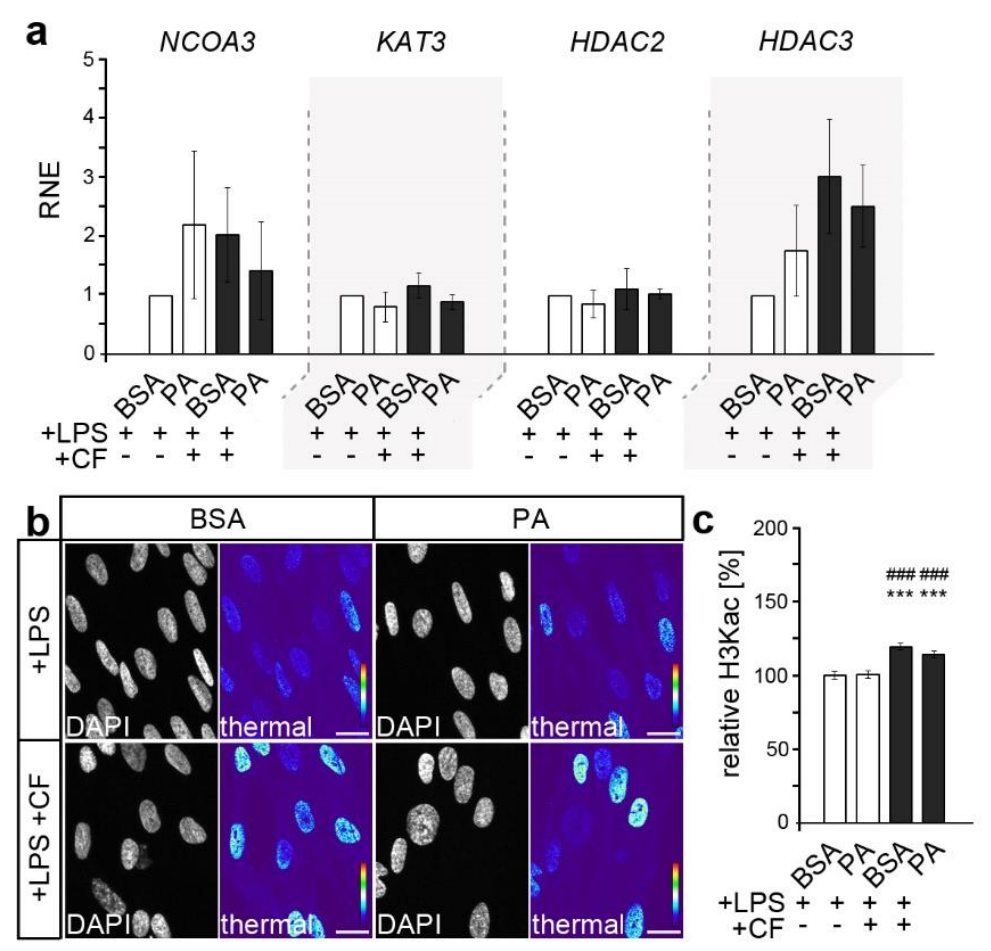

Figure 1: Palmitic acid did not impact H3Kac in compressed HPdLF stimulated with $P$. gingivalis LPS. (a) Quantitative expression analysis of genes encoding histone acetyl transferases NCOA3 and KAT3 and histone deacetylases HDAC2 and HDAC3 in human periodontal ligament fibroblasts (HPdLF) exposed to palmitic acid (PA) and simultaneous stimulated with compressive force (CF) and P. gingivalis-LPS as compared to BSA controls. (b, c) Representative microphotographs of global H3K9/14/18/23/27 acetylation (H3Kac) in HPdLFs treated with PA and co-stimulated with CF and LPS in comparison to BSA controls. Cell nuclei are labeled with DAPI and H3Kac staining intensity is shown in thermal LUT (thermal) analyzed in (c) in relation to BSA control. ${ }^{* * *} \mathrm{p}<0.001$ in relation to BSA+LPS, \#\# $\mathrm{p}<0.001$ in relation to PA+LPS; One-Way ANOVA and post hoc test (Tukey). Scale bars: $10 \mu \mathrm{m}$ in (b). RNE, relative normalized expression.

Considering that PA can also affect repressive histone methylation such as H3K27 trimethylation (H3K27me3) [41,42], we now investigated whether alterations in this modification could contribute to triggering the excessive inflammatory stress response. We 
also performed quantitative PCR on genes encoding components of the polycomb repressive complex 2 (PRC2; EZH1, EZH2, SIN3A, EED), which catalyzes the methylation of H3K27me3 [52]. As shown in Figure 2a, no relevant changes in gene expression were detected, neither due to compressive force nor due to fatty acid exposure. We further analyzed the level of H3K27 trimethylation in dual stimulated HPdLF with quantitative immunofluorescence (Fig. 2b, c). While baseline levels were comparable, additional application of compressive force led to reduced levels of H3K27me3 under fatty acid and control conditions. However, compared to a $82.68 \% \pm 3.66$ reduction in BSA controls, changes in PA cultures $(62.17 \% \pm 4.24)$ were significantly lower ( $\mathrm{p}$-value $\left.0.0276 \times 10^{-2}, * * *\right)$.
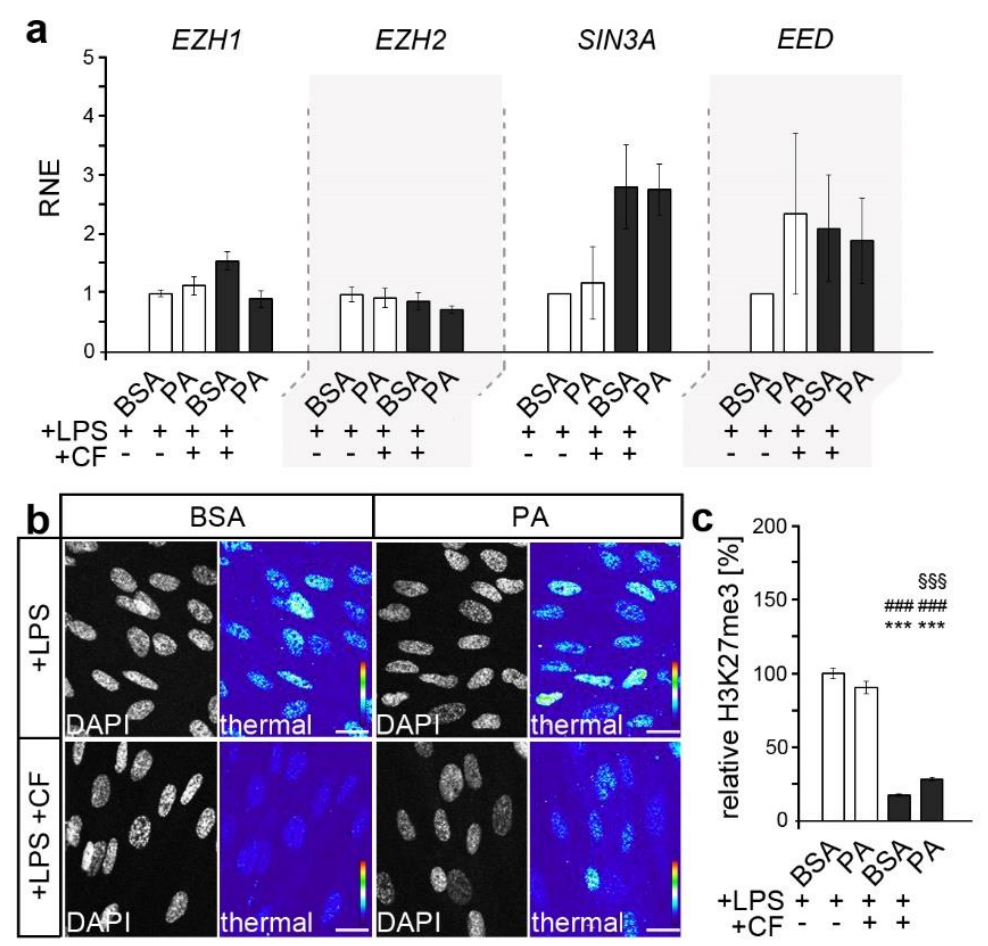

Figure 2: Decrease in H3K27 trimethylation in response to dual stimulation is less in PA-stimulated HPdLFs. (a) Quantitative expression analysis of genes encoding components of the polycomp repressive complex (EZH1, $E Z H 2, S I N 3 A$, and EED) in human periodontal ligament fibroblasts (HPdLF) exposed to palmitic acid (PA) and simultaneous stimulated with compressive force (CF) and P. gingivalis LPS as compared to BSA controls. (b, c) Representative microphotographs of global H3K27me3 (H3K27me3) in HPdLF treated with PA and costimulated with CF and LPS in comparison to BSA controls. Cell nuclei are labeled with DAPI and H3Kac staining intensity is shown in thermal LUT (thermal) analyzed in (c) in relation to BSA control. ${ }^{* * *} \mathrm{p}<0.001$ in relation to BSA+LPS, \#\# $\mathrm{p}<0.001$ in relation to PA+LPS; $\S \S \S \mathrm{p}<0.001$ in relation to BSA+LPS+CF. Scale bars: 10 $\mu \mathrm{m}$ in (b). RNE, relative normalized expression.

3.2. Inhibition of PRC2 enzymes EZH1 and EZH2 abrogates $P A$-induced excessive inflammation, possibly via COX2/PGE2 modulation

To evaluate whether these changes in H3K27me3 level are relevant for the pro-inflammatory effects of PA in dual stimulated HPdLF, we inhibited the core enzymes of the PRC2 EZH1 and EZH2 with UNC1999. To avoid a potential toxicity, we first examined the metabolic activity of HPdLFs treated for one day with different concentrations of UNC1999 (Fig. 3a). This resulted in a slight positive effect of UNC1999; with the highest effect at a concentration of $1.00 \mu \mathrm{M}$.

The activity of H3K27-related histone methyltransferases (HMT) was reduced to $23.23 \% \pm 2.56$ in HPdLF treated for one-day with $1.00 \mu \mathrm{M} \mathrm{UNC1999}$, while lower inhibitor concentrations also resulted in a robust inhibition of HMT activity (Fig. 3b). However, for further analysis, we decided to use $1.00 \mu \mathrm{M}$ UNC1999, which was within the typical range used in previously published in vitro studies $[53,54]$. 
To illustrate the inflammatory processes, we performed a THP1 adhesion assay and analyzed the number of activated THP monocytic cells on stimulated HPdLF (Fig. 3c, d). Comparable to previous results [44], PA exposure led to an enhanced inflammatory response of dual-stimulated HPdLF as compared to BSA controls, even in the presence of DMSO. While the by UNC1999-reduced activity of EZH1 and EZH2 did not alter the number of adherent THP1 cells in the dual-stimulated BSA control, it reduced the excessive activation of monocytic cells in PA cultures to a level comparable to BSA controls.
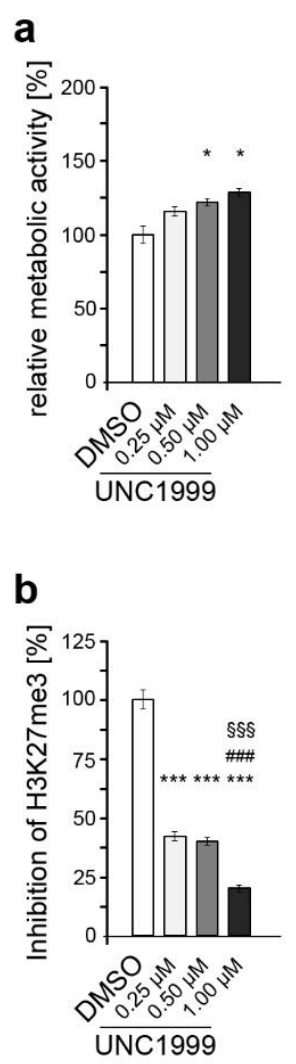

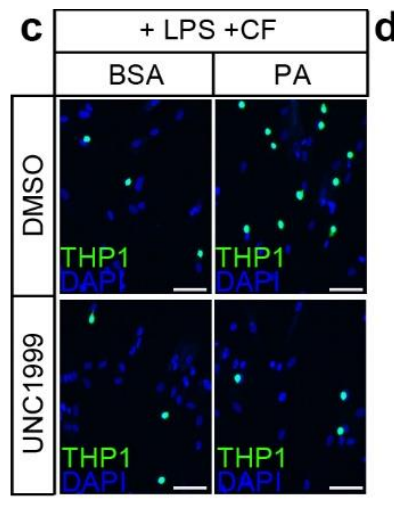

e

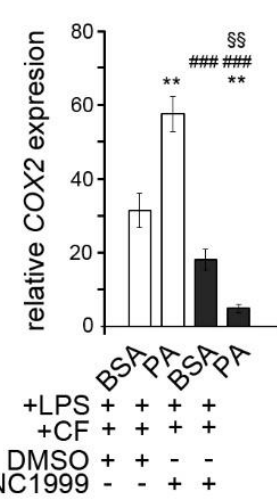

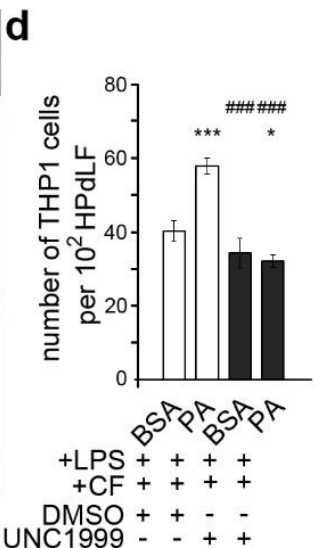

$\mathbf{f}$

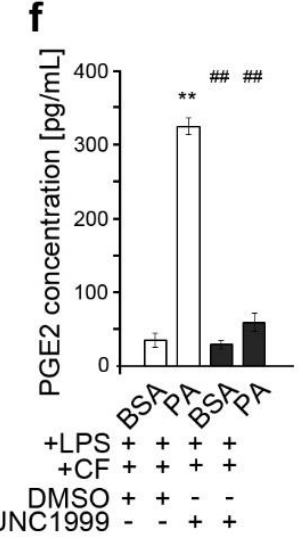

Figure 3: Inhibition of H3K27 trimethylation abrogates the PA-induced excessive inflammatory response of dual stimulated HPdLF. (a) Metabolic activity analyzed in human periodontal ligament fibroblasts (HPdLF) treated with different concentrations of UNC1999 for one day in comparison to DMSO. (b) Analysis of the activity of histone methyltransferases specifically methylating H3K27 in HPdLF treated with $1.00 \mu$ M UNC1999 in comparison to DMSO control. (c, d) Microphotographs of adherent THP1 monocytic cells (green) on compressed (compressive force, $\mathrm{CF}$ ) and $P$. gingivalis LPS-stimulated HPdLFs exposed to palmitic acid (PA) after treatment with $1.00 \mu \mathrm{M}$ UNC1999 in relation to BSA and DMSO controls (c). Cell nuclei were labeled with DAPI (blue). The number of THP1 cells per 102 HPdLF is displayed in (d). (e, f) Analysis of COX2 expression levels (e) and PGE2 secretion (f) in dual stimulated HPdLF exposed to PA after treatment with $1.00 \mu \mathrm{M}$ UNC1999 in comparison to BSA and DMSO controls. ${ }^{*} \mathrm{p}<0.05$ in relation to DMSO in (a) and BSA+LPS+CF+DMSO in (d), ${ }^{* *}$ $\mathrm{p}<0.01$ in relation to BSA+LPS+CF+DMSO in (e) and (f), ${ }^{* * *} \mathrm{p}<0.001$ in relation to DMSO in (b) and $\mathrm{BSA}+\mathrm{LPS}+\mathrm{CF}+\mathrm{DMSO}$ in (d); \#\# $\mathrm{p}<0.01$ in relation to PA+LPS+CF+DMSO; \#\# $\mathrm{p}<0.001$ in relation to DMSO in (b) and PA+LPS+CF+DMSO in (e); $\S \S \mathrm{p}<0.01$ in relation to BSA+LPS+CF+UNC1999. Scale bars: $10 \mu \mathrm{m}$ in (c). 
To further investigate the effects of UNC1999 on inflammatory processes, we determined expression of COX2 (Fig. 3e), a gene encoding an important cytokine that appears to be relevant to the PA-induced excessive inflammatory response of dual stimulated HPdLF [44]. Under DMSO control condition, COX2 expression levels were significantly higher in PA cultures in comparison to BSA controls. This up-regulated COX2 transcription was inhibited by application of UNC1999. To confirm a potential impact of altered COX2-levels on related cytokine secretion, we analyzed PGE2 levels in the supernatant of dual stimulated HPdLFs that were additionally treated with UNC1999 (Fig. 3f). The detected results supported the previously suggested assumption in the context of COX2 expression, as the excessive secretion of PGE2 in dual-stimulated PA cultures was attenuated by UNC1999.

Together, these data suggest that altered reduction of H3K27me3 in dual-stimulated PA cultures may contribute to an excessive inflammatory response of HPdLF, possibly via COX2/PGE2 regulation.

3.2. Palmitic acid causes enhanced trimethylation of H3K27 at Il10 promoter-associated regions in dual stimulated HPdLFs

Because H3K27me3 represses nearby genes, a COX2-associated repressor appeared to be increasingly repressed via that epigenetic modification in dual-stimulated PA cultures. In this context, we next investigated the expression of the anti-inflammatory cytokine IL10 (Fig. 4a), which regulates COX2 transcription [55] and whose expression can be affected by fatty acid-dependent changes in histone modification [51]. We detected a significantly lower IL10 level in dual stimulated PA cultures as compared to BSA controls supporting our hypothesis. Further analysis of the association of trimethylated H3K27 at IL10 promoter regions via chromatin-immunoprecipitation followed by quantitative PCR revealed increased levels of associated H3K27me3 at two IL10 promoter-associated sides in dual-stressed HPdLF exposed to PA (Fig. $4 \mathbf{b}, \mathbf{c}$ ).

In conclusion, enhanced association with trimethylated H3K27 may condition the decreased expression of IL10, which may cause enhanced COX2/PGE signaling in PA exposed HPdLF concomitantly stimulated with $P$. gingivalis-LPS and compressive force.

a
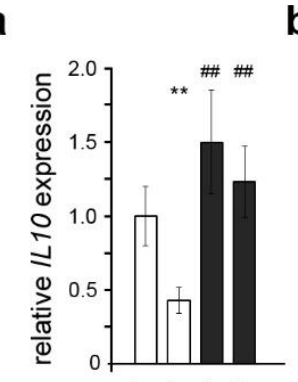

bstrstrp

$+\mathrm{LPS}++++$

DSO ++

UNC1999 ++++ b

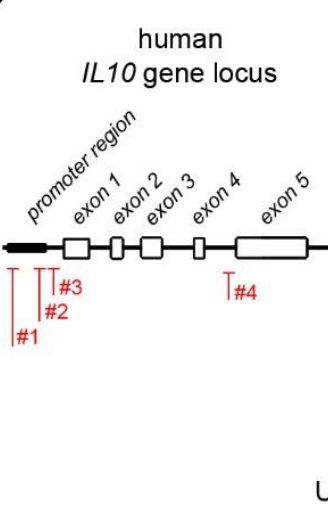

C

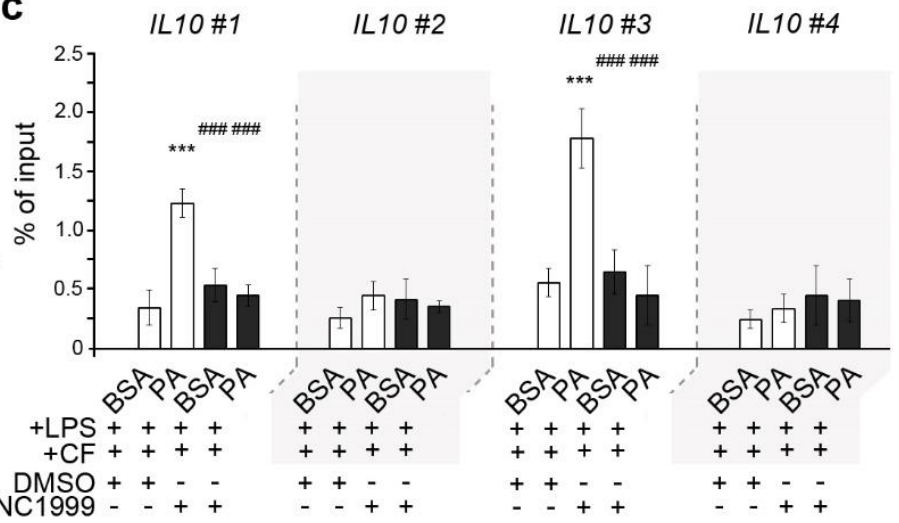

Figure 4: Palmitic acid exposure of dual stressed HPdLF resulted in decreased H3K27 trimethylation near IL10 promoter regions associated with decreased IL10 expression. (a) Quantitative expression analysis of IL10 in human periodontal ligament fibroblasts (HPdLF) exposed to palmitic acid (PA) and simultaneous stimulated with compressive force (CF) and $P$. gingivalisLPS after treatment with $1.00 \mu \mathrm{M}$ UNC1999 compared to BSA and DMSO controls. (b) DNA primer locations in the IL10 gene locus indicating promoter-associated pairs (\#1, \#2 and \#3) and a non-promoter-associated pair (\#4). (c) Quantitative analysis of the association H3K27me3 to specific IL10 gene regions shown in (e) with in dual stimulated HPdLF exposed to PA after UNC1999 treatment in comparison to the respective controls. Data were normalized to the sample input controls. ${ }^{* *} \mathrm{p}<0.01$ in relation to $\mathrm{BSA}+\mathrm{LPS}+\mathrm{CF}+\mathrm{DMSO},{ }^{* * *} \mathrm{p}<0.001$ in relation to $\mathrm{BSA}+\mathrm{LPS}+\mathrm{CF}+\mathrm{DMSO} ; \# \mathrm{p}<0.01$ in relation to PA+LPS+CF+DMSO; \#\# $\mathrm{p}<0.001$ in relation to $\mathrm{PA}+\mathrm{LPS}+\mathrm{CF}+\mathrm{DMSO}$. 


\section{Discussion}

This study investigated the impact of palmitic acid-induced epigenetic changes on the hyper-inflammatory response of HPdLFs that had been simultaneously stimulated with $P$. gingivalis-LPS and compressive force. While the force-induced increase in global H3K acetylation was not affected by PA in LPS-stimulated HPdLFs, reduction of global H3K27 trimethylation was less pronounced in PA cultures. Inhibitor studies suggested an enhanced EZH1/EZH2 activity in dual stimulated PA-exposed HPdLFs as cause for enhanced COX2/PGE2 signaling. Since EZH1/EZH2-modulated H3K27me3 is a repressive mark, increased association at the IL10 promoter provides a possible molecular mechanism via diminished IL10-associated COX2 down-regulation for excessive inflammation in dual stimulated PA cultures.

Epigenetic alterations of pro-inflammatory cytokines are well described for both periodontitis and obesity $[5,56]$. Further, mechanical forces were reported to alter histone marks as well, thus mediating force-dependent gene expression [51,57]. It is therefore likely that epigenetic transcriptional control represents a common level at which mechanical and bacterial influences, as well as obesity-related hyperlipidemic effects, may interfere.

We found force-induced changes in global H3K acetylation and H3K27 trimethylation in LPS-stimulated HPdLF, which, however, were only different for repressive H3K27me3 through PA exposure. This seems surprising, since in compressed HPdLF cultured with monounsaturated oleic acid an increase in global histone acetylation had been detected [51]. However, in this recent study, fibroblasts were not additionally stimulated by $P$. gingivalis-LPS, which has been shown to increase HAT expression in human periodontal ligament stem cells (HPdLSC) [58] and, therefore, minor palmitic acid-induced changes in histone acetylation processes may have been override. This might be due to different pathways that convert lipids to acetyl-CoA, which is necessary for histone acetylation [21,40]. While long-chain fatty acids such as PA need to be actively transported across the mitochondrial membrane where acetyl-CoA conversion takes place [59], shorter fatty acids including pathogenic lipid metabolites could diffuse across those membranes, leading to rapid oxidation to acetyl-CoA [60]. However, it is also known that different fatty acids can have varying influences on histone acylation by regulating distinct enzymes [61].

In accordance with other studies [62,63], we detected reduced global H3K27 trimethylation in HPdLF that were challenged by $P$. gingivalis-LPS and compressive force. However, at least at the RNA level, we could not detect altered expression of the major H3K27-HMT EZH1 and EZH2, which other studies have shown in force-stressed HPdLSC and pathogenically stimulated human B cell lymphoma cells BCBL1 [62,63]. Moreover, we detected increased global H3K27me3 due to PA exposure in dual stressed HPdLF, which is in contradiction to other studies reporting reduced levels of this histone mark [42] or enhanced expression of associated histone demethylases (HMD) such as JMJD3 [64] when stimulated with this fatty acid. This could be due to a variety of reasons including the different cell types and the used PA concentrations as well as the combination of several stimuli possibly leading to different changes in HMT or HMD activity. In this context, posttranslational protein modifications such as acetylation, methylation, phosphorylation, ubiquitination, and O-GlcNAcylation, which can be influenced by various environmental factors [65], were shown to affect the stability and nuclear localization of components of the PRC2 complex as well as its HMT activity and binding of other proteins [66]. Further studies could focus on PA-related changes in the activity of the PRC2 complex to further elucidate the mechanistic background for the increased H3K27me3 level in dual stimulated HPdLF, but this was beyond the scope of our study.

Previously, we reported that PA exposure excessively promoted COX2/PGE2 signaling in $P$. gingivalis LPS stimulated HPdLF that were simultaneously stressed for six hours by compressive force resulting in enhanced activation of monocytic THP1 cells [44]. Similar to our previous study, we detected increased PGE2 secretion in dual-stimulated HPdLF, even when they were additionally cultured with DMSO as an inhibitor control. 
This is consistent with the literature describing increased PGE2 levels in various cells upon both compression and $P$. gingivalis stimulation in addition to fatty acid exposure $[16,67$ $72]$. In this study, we now observed a correlatively increased expression of COX2 in dualstressed HPdLF after PA exposure. This should be taken with caution, as it could also be triggered by DMSO eliciting pro- and anti-inflammatory effects in a cell type-, concentration-, and duration-dependent manner [73]. Nevertheless, both excessive COX2 expression and PGE2 secretion induced by PA exposure in dual-stimulated HPdLF were counterbalanced by UNC1999, which promotes lower H3K27 trimethylation [74]. Transcriptional repression of $C O X 2$ is related to high levels of $\mathrm{H} 3 \mathrm{~K} 27 \mathrm{me} 3$ associated with the gene promoter region, which when removed by EZH2-specific inhibition with DZNep led to increased expression [75]. However, also other epigenetic modifications such as histone acetylation and DNA methylation modulating COX2 transcription highlight a complex regulatory network of this important inflammatory signaling mediator [75,76]. Moreover, we used UNC1999, which also inhibits EZH1 in a concentration-dependent manner [74]. Since EZH1 has similar functions but not fully overlapping targets with EZH2, potential differences could arise from additional inhibition of EZH1. This is also supported by the study of Yamagishi et al., who reported different clusters of regulated genes for both HMTs with a more important role of EZH1 in the inflammatory response [77]. This may also explain why UNC1999 attenuates the excessive activation of THP1 cells to control levels in dual stimulated HPdLF exposed to PA. Future studies may elucidate the specific roles of both H3K27-HMTs in this complex regulation of the COX2/PGE2-driven inflammatory response.

Our study provides evidence that epigenetically modulated IL10-related COX2 regulation is altered in PA-exposed HPdLFs that were concomitantly stressed by mechanical and bacterial stimuli; potentially resulting in this hyper-inflammatory response. The negative regulatory effect of the anti-inflammatory cytokine IL10 on COX2 expression is described comprehensively [55]. Comparable to our results, palmitic acid stimulation of adipocytes resulted in diminished IL10-expression associated with reduced protein secretion [78] further promoting the pro-inflammatory effect of this fatty acid. We detected an enhanced association of trimethylation H3K27 at two position in or close to the IL10 promoter region of dual-stressed HPdLF exposed to PA. Transcriptional repression of IL10 by H3K27 trimethylation is also reported for other cell types, including $\mathrm{T}$ helper cells (Th)0, Th2 and iTreg cells [79] as well as Th17 [80]. In this regard, EZH2 knockdown resulted in increased levels of IL10 [79], which we also observed for gene expression due to pharmacological inhibition with UNC1999. Based on our data, it can be speculated that IL10 is inhibited by a PA-mediated increase in H3K27me3 and, therefore, may fail to limit the COX2/PGE2-driven inflammatory response in dual-stimulated HPdLFs.

Our study is limited for several reasons, including the use of a single fatty acid and LPS as a single pathogenic stimuli, each at one specific concentration, and the study of a single duration of compressive force. Even though the ratio of palmitic acid to BSA has been reported for serum levels of obese patients, in vivo the ratio to other fatty acids are also decisive for hyperlipidemia-related health problems [81-85]. This makes further clinical studies indispensable. After six hours of compressive force one could detect an early phase of inflammatory response, however COX2/PGE2 signaling was reported to be already increased [86,87]. More critical is the remarkable complexity of epigenetic regulation. In this context, changes in one modification require adaptations of other modifications. It has been shown that a reduction of H3K27 trimethylation can lead to increased global and gene-specific levels of activating modifications such as H3K4me3 and H3Kac [88]. However, elucidating these changes in detail was beyond the scope of this study, which aimed to establish a first potential link between the hyper-inflammatory response of dual-stressed hyperlipidemic PdL fibroblasts and the epigenetic regulation of potential key factors. Because of a potential reversibility of this obesity-related adaptation of the inflammatory response, H3K27me3 inhibitors may be of therapeutic interest in addition to the HDAC inhibitors that have already been explored [89]. This is of particular interest considering the increasing frequency of obese patients requiring orthodontic treatment 
and the relevance of periodontal disease in view of associated risks such as tooth root resorption and tooth loss. In the treatment of periodontitis, there are already therapeutic approaches involving modulation of the inflammatory response of the host cells [90]. Epigenetic remodeling may provide a novel platform to overcome unfavorable disease-induced cellular changes by treatment with epigenetic inhibitors during tooth movement.

Author Contributions: Conceptualization, Judit Symmank; Funding acquisition, Judit Symmank; Investigation, Lisa Schuldt, Michael Reimann, Katrin von Brandenstein, Julia Steinmetz, Annika Döding and Judit Symmank; Methodology, Judit Symmank; Project administration, Judit Symmank; Supervision, Collin Jacobs and Judit Symmank; Visualization, Judit Symmank; Writing original draft, Lisa Schuldt, Michael Reimann and Judit Symmank; Writing - review \& editing, Annika Döding, Ulrike Schulze-Späte, Collin Jacobs and Judit Symmank.

Funding: This research was funded by the Interdisciplinary Center of Clinical Research of the Medical Faculty Jena, grant number MSP-08 and the Program for the Support of Third-Party Funding for Young Scientists 2018 Program Line B (Basic) of the Friedrich-Schiller University Jena, grant number DRM/2018-10.

\section{Institutional Review Board Statement: Not applicable}

Informed Consent Statement: Not applicable.

Data Availability Statement: The datasets of this study are available from the corresponding author on reasonable request. The data are not publicly available due to very large size of microscopy images.

Conflicts of Interest: The authors declare no conflict of interest. The funders had no role in the design of the study; in the collection, analyses, or interpretation of data; in the writing of the manuscript, or in the decision to publish the results.

\section{References}

1. Larsson, L., Current concepts of epigenetics and its role in periodontitis. Current oral health reports 2017, 4, $286-293$.

2. Batchelor, P., Is periodontal disease a public health problem? British dental journal 2014, 217, 405-409.

3. Offenbacher, S.; Barros, S.P.; Beck, J.D., Rethinking periodontal inflammation. Journal of periodontology 2008, 79, $1577-1584$.

4. Bird, A., Perceptions of epigenetics. Nature 2007, 447, 396-398.

5. Jurdzinski, K.T.; Potempa, J.; Grabiec, A.M., Epigenetic regulation of inflammation in periodontitis: Cellular mechanisms and therapeutic potential. Clinical epigenetics 2020, 12, 186.

6. Rothbart, S.B.; Strahl, B.D., Interpreting the language of histone and DNA modifications. Biochimica et biophysica acta 2014, 1839, 627-643.

7. Greer, E.L.; Shi, Y., Histone methylation: A dynamic mark in health, disease and inheritance. Nature reviews. Genetics 2012, 13, 343-357.

8. Niederman, R.; Buyle-Bodin, Y.; Lu, B.Y.; Naleway, C.; Robinson, P.; Kent, R., The relationship of gingival crevicular fluid short chain carboxylic acid concentration to gingival inflammation. Journal of clinical periodontology 1996, 23, 743-749.

9. Suganami, T.; Tanimoto-Koyama, K.; Nishida, J.; Itoh, M.; Yuan, X.; Mizuarai, S.; Kotani, H.; Yamaoka, S.; Miyake, K.; Aoe, S., et al., Role of the toll-like receptor 4/nf-kappab pathway in saturated fatty acid-induced inflammatory changes in the interaction between adipocytes and macrophages. Arteriosclerosis, thrombosis, and vascular biology 2007, 27, 84-91.

10. Cooke, A.A.; Connaughton, R.M.; Lyons, C.L.; McMorrow, A.M.; Roche, H.M., Fatty acids and chronic low grade inflammation associated with obesity and the metabolic syndrome. European journal of pharmacology 2016, 785, 207-214.

11. Rossner, S.; Walldius, G.; Bjorvell, H., Fatty acid composition in serum lipids and adipose tissue in severe obesity before and after six weeks of weight loss. Int J Obes 1989, 13, 603-612.

12. Sokolova, M.; Vinge, L.E.; Alfsnes, K.; Olsen, M.B.; Eide, L.; Kaasboll, O.J.; Attramadal, H.; Torp, M.K.; Fosshaug, L.E.; Rashidi, A., et al., Palmitate promotes inflammatory responses and cellular senescence in cardiac fibroblasts. Biochimica et biophysica acta. Molecular and cell biology of lipids 2017, 1862, 234-245.

13. Pillon, N.J.; Azizi, P.M.; Li, Y.E.; Liu, J.; Wang, C.; Chan, K.L.; Hopperton, K.E.; Bazinet, R.P.; Heit, B.; Bilan, P.J., et al., Palmitateinduced inflammatory pathways in human adipose microvascular endothelial cells promote monocyte adhesion and impair insulin transcytosis. American journal of physiology. Endocrinology and metabolism 2015, 309, E35-44.

14. Nemecz, M.; Constantin, A.; Dumitrescu, M.; Alexandru, N.; Filippi, A.; Tanko, G.; Georgescu, A., The distinct effects of palmitic and oleic acid on pancreatic beta cell function: The elucidation of associated mechanisms and effector molecules. Frontiers in pharmacology 2018, 9, 1554 . 
15. Gillet, C.; Spruyt, D.; Rigutto, S.; Dalla Valle, A.; Berlier, J.; Louis, C.; Debier, C.; Gaspard, N.; Malaisse, W.J.; Gangji, V., et al., Oleate abrogates palmitate-induced lipotoxicity and proinflammatory response in human bone marrow-derived mesenchymal stem cells and osteoblastic cells. Endocrinology 2015, 156, 4081-4093.

16. de Souza, C.O.; Valenzuela, C.A.; Baker, E.J.; Miles, E.A.; Rosa Neto, J.C.; Calder, P.C., Palmitoleic acid has stronger antiinflammatory potential in human endothelial cells compared to oleic and palmitic acids. Molecular nutrition $\mathcal{E}$ food research 2018, 62, e1800322.

17. Carrillo, C.; Cavia Mdel, M.; Alonso-Torre, S., Role of oleic acid in immune system; mechanism of action; a review. Nutricion hospitalaria 2012, 27, 978-990.

18. de Lima-Salgado, T.M.; Alba-Loureiro, T.C.; do Nascimento, C.S.; Nunes, M.T.; Curi, R., Molecular mechanisms by which saturated fatty acids modulate tnf-alpha expression in mouse macrophage lineage. Cell biochemistry and biophysics 2011, 59, 8997.

19. Korbecki, J.; Bajdak-Rusinek, K., The effect of palmitic acid on inflammatory response in macrophages: An overview of molecular mechanisms. Inflammation research : official journal of the European Histamine Research Society ... [et al.] 2019, 68, 915-932.

20. Zhou, B.R.; Zhang, J.A.; Zhang, Q.; Permatasari, F.; Xu, Y.; Wu, D.; Yin, Z.Q.; Luo, D., Palmitic acid induces production of proinflammatory cytokines interleukin-6, interleukin-1beta, and tumor necrosis factor-alpha via a nf-kappab-dependent mechanism in hacat keratinocytes. Mediators of inflammation 2013, 2013, 530429.

21. Ferreri, C.; Masi, A.; Sansone, A.; Giacometti, G.; Larocca, A.V.; Menounou, G.; Scanferlato, R.; Tortorella, S.; Rota, D.; Conti, M., et al., Fatty acids in membranes as homeostatic, metabolic and nutritional biomarkers: Recent advancements in analytics and diagnostics. Diagnostics 2016, 7.

22. Amar, S.; Zhou, Q.; Shaik-Dasthagirisaheb, Y.; Leeman, S., Diet-induced obesity in mice causes changes in immune responses and bone loss manifested by bacterial challenge. Proceedings of the National Academy of Sciences of the United States of America 2007, 104, 20466-20471.

23. Nakayama, M.; Ohara, N., Molecular mechanisms of porphyromonas gingivalis-host cell interaction on periodontal diseases. The Japanese dental science review 2017, 53, 134-140.

24. Mulhall, H.; Huck, O.; Amar, S., Porphyromonas gingivalis, a long-range pathogen: Systemic impact and therapeutic implications. Microorganisms 2020, 8.

25. Kato, T.; Yamazaki, K.; Nakajima, M.; Date, Y.; Kikuchi, J.; Hase, K.; Ohno, H.; Yamazaki, K., Oral administration of porphyromonas gingivalis alters the gut microbiome and serum metabolome. mSphere 2018, 3.

26. Arimatsu, K.; Yamada, H.; Miyazawa, H.; Minagawa, T.; Nakajima, M.; Ryder, M.I.; Gotoh, K.; Motooka, D.; Nakamura, S.; Iida, T., et al., Oral pathobiont induces systemic inflammation and metabolic changes associated with alteration of gut microbiota. Scientific reports 2014, 4, 4828.

27. Cani, P.D.; Amar, J.; Iglesias, M.A.; Poggi, M.; Knauf, C.; Bastelica, D.; Neyrinck, A.M.; Fava, F.; Tuohy, K.M.; Chabo, C., et al., Metabolic endotoxemia initiates obesity and insulin resistance. Diabetes 2007, 56, 1761-1772.

28. Le Sage, F.; Meilhac, O.; Gonthier, M.P., Porphyromonas gingivalis lipopolysaccharide induces pro-inflammatory adipokine secretion and oxidative stress by regulating toll-like receptor-mediated signaling pathways and redox enzymes in adipocytes. Molecular and cellular endocrinology 2017, 446, 102-110.

29. Jonsson, D.; Nebel, D.; Bratthall, G.; Nilsson, B.O., The human periodontal ligament cell: A fibroblast-like cell acting as an immune cell. J Periodontal Res 2011, 46, 153-157.

30. Proff, P.; Reicheneder, C.; Faltermeier, A.; Kubein-Meesenburg, D.; Romer, P., Effects of mechanical and bacterial stressors on cytokine and growth-factor expression in periodontal ligament cells. Journal of orofacial orthopedics= Fortschritte der Kieferorthopadie : Organ/official journal Deutsche Gesellschaft fur Kieferorthopadie 2014, 75, 191-202.

31. Yamaguchi, M.; Fukasawa, S., Is inflammation a friend or foe for orthodontic treatment?: Inflammation in orthodontically induced inflammatory root resorption and accelerating tooth movement. International journal of molecular sciences $2021,22$.

32. Weltman, B.; Vig, K.W.; Fields, H.W.; Shanker, S.; Kaizar, E.E., Root resorption associated with orthodontic tooth movement: A systematic review. American journal of orthodontics and dentofacial orthopedics : official publication of the American Association of Orthodontists, its constituent societies, and the American Board of Orthodontics 2010, 137, 462-476; discussion 412A.

33. Wishney, M., Potential risks of orthodontic therapy: A critical review and conceptual framework. Australian dental journal 2017, 62 Suppl 1, 86-96.

34. Kirschneck, C.; Fanghanel, J.; Wahlmann, U.; Wolf, M.; Roldan, J.C.; Proff, P., Interactive effects of periodontitis and orthodontic tooth movement on dental root resorption, tooth movement velocity and alveolar bone loss in a rat model. Annals of anatomy= Anatomischer Anzeiger : official organ of the Anatomische Gesellschaft 2017, 210, 32-43.

35. Jayachandran, T.; Srinivasan, B.; Padmanabhan, S., Salivary leptin levels in normal weight and overweight individuals and their correlation with orthodontic tooth movement. The Angle orthodontist 2017, 87, 739-744.

36. Michelogiannakis, D.; Rossouw, P.E.; Khan, J.; Akram, Z.; Menenakos, E.; Javed, F., Influence of increased body mass index on orthodontic tooth movement and related parameters in children and adolescents: A systematic review of longitudinal controlled clinical studies. Journal of orthodontics 2019, 46, 323-334.

37. Saloom, H.F.; Papageorgiou, S.N.; Carpenter, G.H.; Cobourne, M.T., Impact of obesity on orthodontic tooth movement in adolescents: A prospective clinical cohort study. Journal of dental research 2017, 96, 547-554. 
38. Consolaro, A., Obesity and orthodontic treatment: Is there any direct relationship? Dental press journal of orthodontics 2017, 22, 21-25.

39. Ramos-Lopez, O.; Milagro, F.I.; Riezu-Boj, J.I.; Martinez, J.A., Epigenetic signatures underlying inflammation: An interplay of nutrition, physical activity, metabolic diseases, and environmental factors for personalized nutrition. Inflammation research : official journal of the European Histamine Research Society ... [et al.] 2021, 70, 29-49.

40. McDonnell, E.; Crown, S.B.; Fox, D.B.; Kitir, B.; Ilkayeva, O.R.; Olsen, C.A.; Grimsrud, P.A.; Hirschey, M.D., Lipids reprogram metabolism to become a major carbon source for histone acetylation. Cell reports 2016, 17, 1463-1472.

41. Pascual, G.; Dominguez, D.; Elosua-Bayes, M.; Beckedorff, F.; Laudanna, C.; Bigas, C.; Douillet, D.; Greco, C.; Symeonidi, A.; Hernandez, I., et al., Dietary palmitic acid promotes a prometastatic memory via schwann cells. Nature 2021, 599, 485-490.

42. Kumar, S.; Pamulapati, H.; Tikoo, K., Fatty acid induced metabolic memory involves alterations in renal histone h3k36me2 and h3k27me3. Molecular and cellular endocrinology 2016, 422, 233-242.

43. Heerboth, S.; Lapinska, K.; Snyder, N.; Leary, M.; Rollinson, S.; Sarkar, S., Use of epigenetic drugs in disease: An overview. Genetics E epigenetics 2014, 6, 9-19.

44. Symmank, J.; Appel, S.; Bastian, J.A.; Knaup, I.; Marciniak, J.; Hennig, C.L.; Doding, A.; Schulze-Spate, U.; Jacobs, C.; Wolf, M., Hyperlipidemic conditions impact force-induced inflammatory response of human periodontal ligament fibroblasts concomitantly challenged with p. Gingivalis-lps. International journal of molecular sciences 2021, 22.

45. Greenhough, A.; Smartt, H.J.; Moore, A.E.; Roberts, H.R.; Williams, A.C.; Paraskeva, C.; Kaidi, A., The cox-2/pge2 pathway: Key roles in the hallmarks of cancer and adaptation to the tumour microenvironment. Carcinogenesis 2009, 30, 377-386.

46. Kirschneck, C.; Batschkus, S.; Proff, P.; Kostler, J.; Spanier, G.; Schroder, A., Valid gene expression normalization by rt-qpcr in studies on hpdl fibroblasts with focus on orthodontic tooth movement and periodontitis. Scientific reports 2017, 7, 14751.

47. Symmank, J.; Chorus, M.; Appel, S.; Marciniak, J.; Knaup, I.; Bastian, A.; Hennig, C.L.; Doding, A.; Schulze-Spate, U.; Jacobs, C., et al., Distinguish fatty acids impact survival, differentiation and cellular function of periodontal ligament fibroblasts. Scientific reports 2020, 10, 15706.

48. Stemmler, A.; Symmank, J.; Steinmetz, J.; von Brandenstein, K.; Hennig, C.L.; Jacobs, C., Gdf15 supports the inflammatory response of pdl fibroblasts stimulated by $\mathrm{p}$. Gingivalis lps and concurrent compression. International journal of molecular sciences 2021, 22.

49. Symmank, J.; Bayer, C.; Reichard, J.; Pensold, D.; Zimmer-Bensch, G., Neuronal lhx1 expression is regulated by dnmt1dependent modulation of histone marks. Epigenetics 2020, 15, 1259-1274.

50. Haring, M.; Offermann, S.; Danker, T.; Horst, I.; Peterhansel, C.; Stam, M., Chromatin immunoprecipitation: Optimization, quantitative analysis and data normalization. Plant methods 2007, 3, 11.

51. Schuldt, L.; von Brandenstein, K.; Jacobs, C.; Symmank, J., Oleic acid-related anti-inflammatory effects in force-stressed pdl fibroblasts are mediated by h3 lysine acetylation associated with altered il10 expression. BioRxiv Preprint Server 2022.

52. Margueron, R.; Reinberg, D., The polycomb complex prc2 and its mark in life. Nature 2011, 469, 343-349.

53. Grinshtein, N.; Rioseco, C.C.; Marcellus, R.; Uehling, D.; Aman, A.; Lun, X.; Muto, O.; Podmore, L.; Lever, J.; Shen, Y., et al., Small molecule epigenetic screen identifies novel ezh2 and hdac inhibitors that target glioblastoma brain tumor-initiating cells. Oncotarget 2016, 7, 59360-59376.

54. Katona, B.W.; Liu, Y.; Ma, A.; Jin, J.; Hua, X., Ezh2 inhibition enhances the efficacy of an egfr inhibitor in suppressing colon cancer cells. Cancer biology $\mathcal{E}$ therapy 2014, 15, 1677-1687.

55. Berg, D.J.; Zhang, J.; Lauricella, D.M.; Moore, S.A., Il-10 is a central regulator of cyclooxygenase-2 expression and prostaglandin production. Journal of immunology 2001, 166, 2674-2680.

56. Jung, B.C.; Kang, S., Epigenetic regulation of inflammatory factors in adipose tissue. Biochimica et biophysica acta. Molecular and cell biology of lipids 2021, 1866, 159019.

57. Le, H.Q.; Ghatak, S.; Yeung, C.Y.; Tellkamp, F.; Gunschmann, C.; Dieterich, C.; Yeroslaviz, A.; Habermann, B.; Pombo, A.; Niessen, C.M., et al., Mechanical regulation of transcription controls polycomb-mediated gene silencing during lineage commitment. Nature cell biology 2016, 18, 864-875.

58. Diomede, F.; Thangavelu, S.R.; Merciaro, I.; D'Orazio, M.; Bramanti, P.; Mazzon, E.; Trubiani, O., Porphyromonas gingivalis lipopolysaccharide stimulation in human periodontal ligament stem cells: Role of epigenetic modifications to the inflammation. European journal of histochemistry : EJH 2017, 61, 2826.

59. Schwenk, R.W.; Holloway, G.P.; Luiken, J.J.; Bonen, A.; Glatz, J.F., Fatty acid transport across the cell membrane: Regulation by fatty acid transporters. Prostaglandins, leukotrienes, and essential fatty acids 2010, 82, 149-154.

60. Kamp, F.; Hamilton, J.A., How fatty acids of different chain length enter and leave cells by free diffusion. Prostaglandins, leukotrienes, and essential fatty acids 2006, 75, 149-159.

61. Dimri, M.; Bommi, P.V.; Sahasrabuddhe, A.A.; Khandekar, J.D.; Dimri, G.P., Dietary omega-3 polyunsaturated fatty acids suppress expression of ezh2 in breast cancer cells. Carcinogenesis 2010, 31, 489-495.

62. Yu, X.; Shahir, A.M.; Sha, J.; Feng, Z.; Eapen, B.; Nithianantham, S.; Das, B.; Karn, J.; Weinberg, A.; Bissada, N.F., et al., Shortchain fatty acids from periodontal pathogens suppress histone deacetylases, ezh2, and suv39h1 to promote kaposi's sarcomaassociated herpesvirus replication. Journal of virology 2014, 88, 4466-4479. 
63. Li, Q.; Sun, X.; Tang, Y.; Qu, Y.; Zhou, Y.; Zhang, Y., Ezh2 reduction is an essential mechanoresponse for the maintenance of super-enhancer polarization against compressive stress in human periodontal ligament stem cells. Cell death $\mathcal{E}$ disease 2020, 11, 757.

64. Davis, F.M.; denDekker, A.; Joshi, A.D.; Wolf, S.J.; Audu, C.; Melvin, W.J.; Mangum, K.; Riordan, M.O.; Kunkel, S.L.; Gallagher, K.A., Palmitate-tlr4 signaling regulates the histone demethylase, jmjd3, in macrophages and impairs diabetic wound healing. European journal of immunology 2020, 50, 1929-1940.

65. Ramazi, S.; Zahiri, J., Posttranslational modifications in proteins: Resources, tools and prediction methods. Database : the journal of biological databases and curation 2021, 2021.

66. Yang, Y.; Li, G., Post-translational modifications of prc2: Signals directing its activity. Epigenetics $\mathcal{E}$ chromatin $2020,13,47$.

67. Arisaka, M.; Arisaka, O.; Yamashiro, Y., Fatty acid and prostaglandin metabolism in children with diabetes mellitus. Ii. The effect of evening primrose oil supplementation on serum fatty acid and plasma prostaglandin levels. Prostaglandins, leukotrienes, and essential fatty acids 1991, 43, 197-201.

68. Coll, T.; Palomer, X.; Blanco-Vaca, F.; Escola-Gil, J.C.; Sanchez, R.M.; Laguna, J.C.; Vazquez-Carrera, M., Cyclooxygenase 2 inhibition exacerbates palmitate-induced inflammation and insulin resistance in skeletal muscle cells. Endocrinology 2010, 151, 537-548.

69. Huang, S.K.; White, E.S.; Wettlaufer, S.H.; Grifka, H.; Hogaboam, C.M.; Thannickal, V.J.; Horowitz, J.C.; Peters-Golden, M., Prostaglandin e(2) induces fibroblast apoptosis by modulating multiple survival pathways. FASEB journal : official publication of the Federation of American Societies for Experimental Biology 2009, 23, 4317-4326.

70. Seifi, M.; Eslami, B.; Saffar, A.S., The effect of prostaglandin e2 and calcium gluconate on orthodontic tooth movement and root resorption in rats. Eur J Orthod 2003, 25, 199-204.

71. Saito, M.; Saito, S.; Ngan, P.W.; Shanfeld, J.; Davidovitch, Z., Interleukin 1 beta and prostaglandin e are involved in the response of periodontal cells to mechanical stress in vivo and in vitro. American journal of orthodontics and dentofacial orthopedics : official publication of the American Association of Orthodontists, its constituent societies, and the American Board of Orthodontics 1991, 99, 226240.

72. Hageman, J.R.; McCulloch, K.; Hunt, C.E.; Cobb, M.; Quade, B.; Crussi, F.; Pachman, L., Oleic acid lung injury increases plasma prostaglandin levels. Prostaglandins, leukotrienes, and essential fatty acids 1989, 35, 157-164.

73. Hollebeeck, S.; Raas, T.; Piront, N.; Schneider, Y.J.; Toussaint, O.; Larondelle, Y.; During, A., Dimethyl sulfoxide (dmso) attenuates the inflammatory response in the in vitro intestinal caco-2 cell model. Toxicology letters 2011, 206, $268-275$.

74. Konze, K.D.; Ma, A.; Li, F.; Barsyte-Lovejoy, D.; Parton, T.; Macnevin, C.J.; Liu, F.; Gao, C.; Huang, X.P.; Kuznetsova, E., et al., An orally bioavailable chemical probe of the lysine methyltransferases ezh2 and ezh1. ACS chemical biology 2013, 8, $1324-1334$.

75. Coward, W.R.; Feghali-Bostwick, C.A.; Jenkins, G.; Knox, A.J.; Pang, L., A central role for g9a and ezh2 in the epigenetic silencing of cyclooxygenase-2 in idiopathic pulmonary fibrosis. FASEB journal : official publication of the Federation of American Societies for Experimental Biology 2014, 28, 3183-3196.

76. Coward, W.R.; Watts, K.; Feghali-Bostwick, C.A.; Knox, A.; Pang, L., Defective histone acetylation is responsible for the diminished expression of cyclooxygenase 2 in idiopathic pulmonary fibrosis. Molecular and cellular biology 2009, 29, $4325-4339$.

77. Yamagishi, M.; Hori, M.; Fujikawa, D.; Ohsugi, T.; Honma, D.; Adachi, N.; Katano, H.; Hishima, T.; Kobayashi, S.; Nakano, K., et al., Targeting excessive ezh1 and ezh2 activities for abnormal histone methylation and transcription network in malignant lymphomas. Cell reports 2019, 29, 2321-2337 e2327.

78. Bradley, R.L.; Fisher, F.F.; Maratos-Flier, E., Dietary fatty acids differentially regulate production of tnf-alpha and il-10 by murine 3t3-11 adipocytes. Obesity 2008, 16, 938-944.

79. Zhang, Y.; Kinkel, S.; Maksimovic, J.; Bandala-Sanchez, E.; Tanzer, M.C.; Naselli, G.; Zhang, J.G.; Zhan, Y.; Lew, A.M.; Silke, J., et al., The polycomb repressive complex 2 governs life and death of peripheral t cells. Blood 2014, 124, 737-749.

80. Escobar, T.M.; Kanellopoulou, C.; Kugler, D.G.; Kilaru, G.; Nguyen, C.K.; Nagarajan, V.; Bhairavabhotla, R.K.; Northrup, D.; Zahr, R.; Burr, P., et al., Mir-155 activates cytokine gene expression in th17 cells by regulating the DNA-binding protein jarid2 to relieve polycomb-mediated repression. Immunity 2014, 40, 865-879.

81. Bierman, E.L.; Dole, V.P.; Roberts, T.N., An abnormality of nonesterified fatty acid metabolism in diabetes mellitus. Diabetes 1957, 6, 475-479.

82. Taskinen, M.R.; Bogardus, C.; Kennedy, A.; Howard, B.V., Multiple disturbances of free fatty acid metabolism in noninsulindependent diabetes. Effect of oral hypoglycemic therapy. The Journal of clinical investigation 1985, 76, 637-644.

83. Soares, E.A.; Nakagaki, W.R.; Garcia, J.A.; Camilli, J.A., Effect of hyperlipidemia on femoral biomechanics and morphology in low-density lipoprotein receptor gene knockout mice. Journal of bone and mineral metabolism 2012, 30, 419-425.

84. Kissebah, A.H.; Alfarsi, S.; Adams, P.W.; Wynn, V., Role of insulin resistance in adipose tissue and liver in the pathogenesis of endogenous hypertriglyceridaemia in man. Diabetologia 1976, 12, 563-571.

85. Cistola, D.P.; Small, D.M., Fatty acid distribution in systems modeling the normal and diabetic human circulation. A $13 \mathrm{c}$ nuclear magnetic resonance study. The Journal of clinical investigation 1991, 87, 1431-1441.

86. Li, Y.; Li, M.; Tan, L.; Huang, S.; Zhao, L.; Tang, T.; Liu, J.; Zhao, Z., Analysis of time-course gene expression profiles of a periodontal ligament tissue model under compression. Archives of oral biology 2013, 58, 511-522. 
87. Kanzaki, H.; Chiba, M.; Shimizu, Y.; Mitani, H., Periodontal ligament cells under mechanical stress induce osteoclastogenesis by receptor activator of nuclear factor kappab ligand up-regulation via prostaglandin e2 synthesis. Journal of bone and mineral research : the official journal of the American Society for Bone and Mineral Research 2002, 17, 210-220.

88. Pande, V., Understanding the complexity of epigenetic target space. Journal of medicinal chemistry 2016, 59, 1299-1307.

89. Cantley, M.D.; Bartold, P.M.; Marino, V.; Fairlie, D.P.; Le, G.T.; Lucke, A.J.; Haynes, D.R., Histone deacetylase inhibitors and periodontal bone loss. J Periodontal Res 2011, 46, 697-703.

90. Yang, B.; Pang, X.; Li, Z.; Chen, Z.; Wang, Y., Immunomodulation in the treatment of periodontitis: Progress and perspectives. Frontiers in immunology 2021, 12, 781378. 